ARTICLE

Received 19 Jun 2015 | Accepted 14 Sep 2015 | Published 9 Nov 2015

DOI: $10.1038 /$ ncomms 9631

\title{
Atomic and vibrational origins of mechanical toughness in bioactive cement during setting
}

\author{
Kun V. Tian', Bin Yang ${ }^{2,3}$, Yuanzheng Yue ${ }^{4,5}$, Daniel T. Bowron ${ }^{6}$, Jerry Mayers ${ }^{6}$, Robert S. Donnan ${ }^{3}$, \\ Csaba Dobó-Nagy ${ }^{1}$, John W. Nicholson?7, De-Cai Fang ${ }^{8}$, A. Lindsay Greer ${ }^{9}$, Gregory A. Chass ${ }^{10}$ \\ \& G. Neville Greaves $4,9,11$
}

Bioactive glass ionomer cements (GICs) have been in widespread use for $\sim 40$ years in dentistry and medicine. However, these composites fall short of the toughness needed for permanent implants. Significant impediment to improvement has been the requisite use of conventional destructive mechanical testing, which is necessarily retrospective. Here we show quantitatively, through the novel use of calorimetry, terahertz $(\mathrm{THz})$ spectroscopy and neutron scattering, how GIC's developing fracture toughness during setting is related to interfacial $\mathrm{THz}$ dynamics, changing atomic cohesion and fluctuating interfacial configurations. Contrary to convention, we find setting is non-monotonic, characterized by abrupt features not previously detected, including a glass-polymer coupling point, an early setting point, where decreasing toughness unexpectedly recovers, followed by stress-induced weakening of interfaces. Subsequently, toughness declines asymptotically to long-term fracture test values. We expect the insight afforded by these in situ non-destructive techniques will assist in raising understanding of the setting mechanisms and associated dynamics of cementitious materials.

\footnotetext{
${ }^{1}$ Department of Oral Diagnostics, Faculty of Dentistry, Semmelweis University, Budapest 1088, Hungary. ${ }^{2}$ Department of Electronic and Electrical Engineering, University of Chester, Thornton Science Park, Chester CH2 4NU, UK. ${ }^{3}$ School of Electronic Engineering and Computer Science, Queen Mary University of London, London E1 4NS, UK. ${ }^{4}$ Laboratory of Extreme Glassy State, State Key Laboratory of Silicate Materials for Architectures, Wuhan University of Technology, Wuhan 430070, China. ${ }^{5}$ Department of Chemistry and Bioscience, Aalborg University, DK-9220 Aalborg, Denmark. ${ }^{6}$ ISIS Facility, STFC Rutherford Appleton Laboratory, Chilton, Didcot, Oxon OX11 OQX, UK. ${ }^{7}$ School of Sport, Health and Applied Science, St Mary's University, London TW1 4SX, UK. ${ }^{8}$ College of Chemistry, Beijing Normal University, Beijing 100875, China. ${ }^{9}$ Department of Materials Science and Metallurgy, University of Cambridge, 27 Charles Babbage Road, Cambridge CB3 OFS, UK. ${ }^{10}$ School of Biological and Chemical Sciences, Queen Mary University of London, London E1 4NS, UK. ${ }^{11}$ Department of Physics, Institute of Mathematics, Physics and Computer Science, Aberystwyth University, Aberystwyth SY23 3BZ, UK. Correspondence and requests for materials should be addressed to G.A.C. (email: g.chass@qmul.ac.uk) or to G.N.G. (email: gng25@cam.ac.uk).
} 
W orldwide demand for durable biomaterials emanates from population ageing and from emergent developing countries. Historically, the implantation of foreign materials into the body has been dentistry led. However, the optimisation of tooth replacements remains incomplete, problems stemming from conflicts between mechanical toughness, biocompatibility, adhesion and appearance. For commonly used mercury-silver amalgams, this is compounded by toxicity and disposal. The United Nations Environment Programme assesses mercury to be 'a global threat to human and environmental health,' listing amalgams as a source ${ }^{1}$. With 125 million amalgam restorations carried out annually in Europe, the European Commission advocates atraumatic restorative treatment using mercury-free alternatives ${ }^{2}$, highlighting glass ionomer cements (GICs) (Fig. 1a) as an excellent option. Developed over 40 years ${ }^{3-8}$, GICs are the product of a basic fluoro-phospho-alumino-silicate glass powder and an aqueous poly(acrylic) acid (PAA) solution (Fig. 1b; Methods)-also known as glass polyalkenoate cements ${ }^{9}$. Although cost-effective and environmentally friendly ${ }^{2}$, caries-resistant and bioactively mineralizing dentine $e^{10,11}$, GICs remain too brittle for permanent implants ${ }^{12,13}$. With exceptional bonding to the apatite phase of bone, GICs have also been considered for other aspects of surgery ${ }^{14-16}$, but necessarily confined to non-load-bearing applications with moderate durability requirements ${ }^{17}$.
Damage tolerance is assessed through fracture toughness $K_{\mathrm{C}}$ and yield strength $\sigma_{\mathrm{Y}}$, both traditionally measured by destructive methods ${ }^{18}$. Average values for dental materials and their components have been collated to create Fig. 1c. This $\log K_{\mathrm{C}}$ versus $\log \sigma_{\mathrm{Y}}$ plot follows the Ashby scheme ${ }^{19}$ widely used elsewhere in mechanics to categorize conflicts between strength and toughness in composite materials ${ }^{20}$. Polymers and ceramics lie in the lower half with $K_{\mathrm{C}}$ values greater than $1 \mathrm{MPa} \mathrm{m}^{1 / 2}$; brittle materials such as glasses have $K_{\mathrm{C}}$ values less than this. Indeed the yield strength $\sigma_{\mathrm{Y}}$ of glassy materials covers many decades ${ }^{21}$ from $\sim 10 \mathrm{GPa}$ (glass fibres) to $\sim 1 \mathrm{MPa}$ (pre-damaged glass). Respective $K_{\mathrm{C}}$ and $\sigma_{\mathrm{Y}}$ values of GICs cluster around those of dentine and amalgam, but clearly have less toughness and strength than either.

For composites in general, and GICs in particular, $K_{\mathrm{C}}$ and $\sigma_{\mathrm{Y}}$ develop during setting, starting out as highly deformable and incompressible slurries that subsequently harden to form rigid, inflexible cements. The compressibility $\kappa$ relates directly to the shape of the interatomic potentials of a given system ${ }^{22}$. The narrower and deeper the potential is, the stronger and more rigid the atomic cohesion and vice versa. More rigid materials have a higher shear modulus $G$ and lower $\kappa$, but most importantly tend to have a higher $G \cdot \kappa$, and to be brittle. In particular, Poisson's ratio $v$ (ref. 22), a function of $G \cdot \kappa$, sharply differentiates between brittleness and ductility. If toughness is converted to fracture a

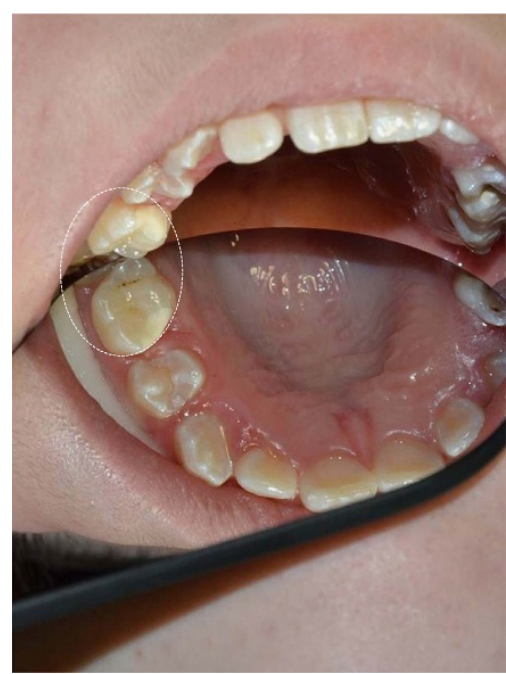

C

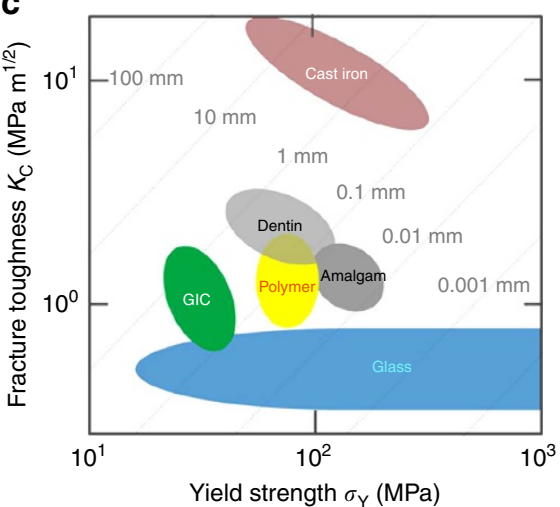

b

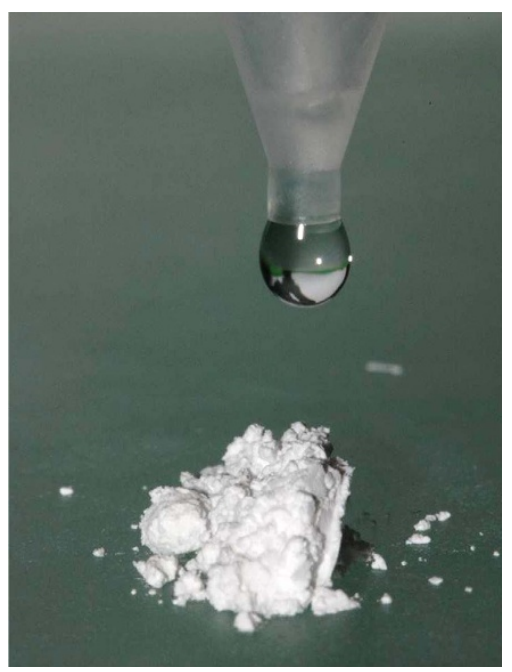

d

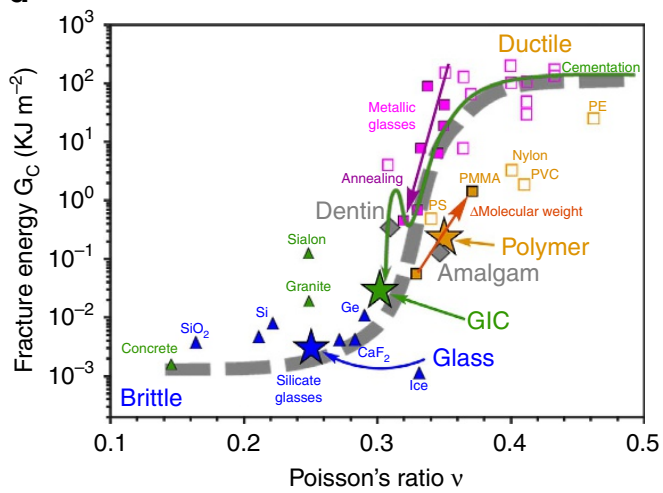

Figure 1 | GICs properties and glass nanoscopic structure. (a) GIC restorative, including occlusal view. (b) G338 fluoro-alumino-silicate glass powder and dangling aqueous polymer (acrylic acid). (c) Fracture toughness $K_{C}$ versus strength $\sigma_{Y}$ for dental materials compiled as an Ashby plot ${ }^{19}$ : dentin, glass and polymers ${ }^{20,21,45}, \mathrm{GICs}^{46,47}$ and amalgam ${ }^{60}$. (d) Fracture energy $G_{c}$ versus Poisson's ratio $v$ and the brittle-ductile transition ${ }^{22,23}$, expanded for a wide range of materials; $v$ values for dental materials ${ }^{24,25,61,62}$ combined with $G_{C}$ values ${ }^{20-23,45-47,60,61}$; toughness decline during setting indicated by the dashed arrow; trends for annealing and polymerization (solid arrows). a and $\mathbf{b}$ courtesy Faculty of Dentistry, Semmelweis University. 
energy $G_{\mathrm{C}}$ (fracture energy $G_{\mathrm{C}}$ is related to fracture toughness $K_{\mathrm{C}}$ by $G_{C}=K_{C}^{2}\left(1-v^{2}\right) / E$, where $E$ is Young's modulus and $v$ is Poisson's ratio), as a function of $v$, this forms a clear sigmoid, with an inflection point (at $v \approx 0.33)^{23}$ separating ductile $(v>\sim 0.33)$ from brittle $(v<\sim 0.33)$ materials (Fig. 1d). Originally discovered for metals ${ }^{23}$, this important empirical relationship also holds for inorganic glasses and polymers, the ingredients of GICs and other non-metals. All these materials have been incorporated into Fig. $1 \mathrm{~d}$ to create a guide for assessing the extent of brittleness of dental materials. In particular, as setting advances, $v$ must decrease significantly, from $v \approx 0.5$, when the cement is a virtually incompressible liquid to $v<\sim 0.33$, as it solidifies into a brittle solid. This is illustrated by the dashed arrow for GICs, which finally become too brittle, with $v \approx 0.30$ (ref. 24) compared with amalgam with $v \approx 0.34$ (refs 22,25). The ultimate aim is to modify GICs so that they are closer in toughness to amalgam and dentin.

The initial setting mechanism of GICs is an acid-base reaction between the aqueous PAA and the glass component (Fig. 1b; Methods) $)^{3,4}$. As with alumina-silicate glasses, water corrosion ruptures bridging oxygens (BOs) to form $\mathrm{SiOH}$ (ref. 26) and $\mathrm{AlOH}$ (ref. 27) groups, initially creating an aqueous gel at the glass surface. For the $\mathrm{G} 338$ glass used in GICs, $\mathrm{PO}_{4}^{-}$and $\mathrm{F}^{-}$will also be released, along with $\mathrm{Na}^{+}$cations, freeing $\mathrm{Al}^{3+}$ and $\mathrm{Ca}^{2+}$ cations to crosslink the polymer to form a strong polysalt matrix $^{4,5}$. Aluminium chelation by the polymer drives the conversion of $\mathrm{Al}(\mathrm{IV})$ tetrahedra to higher-coordinated sites ${ }^{28}$ at the interfaces between glass and the matrix, as well as those between crosslinked polymer chains within the matrix.
Higher-coordinated sites include both pyramidal $\mathrm{Al}(\mathrm{V})$ as well as octahedral $\mathrm{Al}(\mathrm{VI})$ geometries. Similar changes in interfacial configuration in proteins, for example, are manifested by variations of orientation-dependent dynamics in the sub- $\mathrm{THz}$ range ${ }^{29}$. These low-frequency modes are known to modulate mechanical $^{30}$, optical ${ }^{31}$ and biophysical properties ${ }^{32}$ of macromolecular systems.

We have therefore turned to non-destructive techniques that record changing atomic structures, associated collective sub- $\mathrm{THz}$ dynamics and atomic cohesion during the first 3 days of setting, uncovering highly nonlinear behaviour over various stages, and providing indications of the sources of eventual brittleness and low strength.

\section{Results}

Heterogeneous glass. To make our extended in situ experiments relevant to current dental practice, we have chosen the commercial G338 ionomer glass (Fig. 1b), in regular use since the early 1980s. To ensure practical relevance, we employed standard clinical preparatory mixing to examine setting from the polymerglass mixture to the hardened cement. The glass powder has been imaged by transmission electron microscopy (TEM) and exhibits significant heterogeneity, with three glass phases (GPs) identifiable on the scale of $5-50 \mathrm{~nm}$ (Fig. 2a). Figure $2 \mathrm{~b}$ and Supplementary Fig. 1 show the isobaric heat capacity $C_{\mathrm{p}}$ of both G338 and GIC samples as a function of temperature determined by differential scanning calorimetry (DSC). The $C_{\mathrm{p}}$ of fresh G338 sample (red curve) exhibits the effects of water loss, after which (blue curve) three sharp glass transitions can be deciphered.

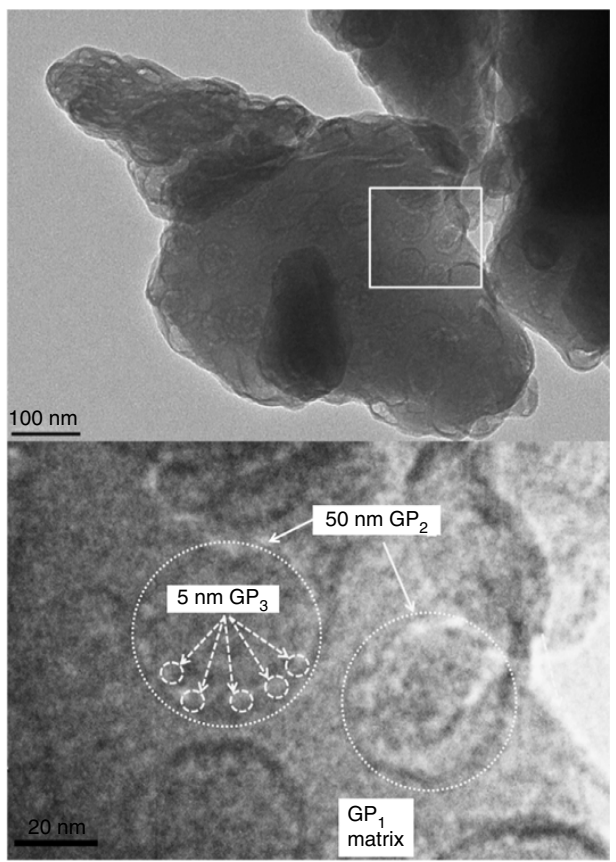

b
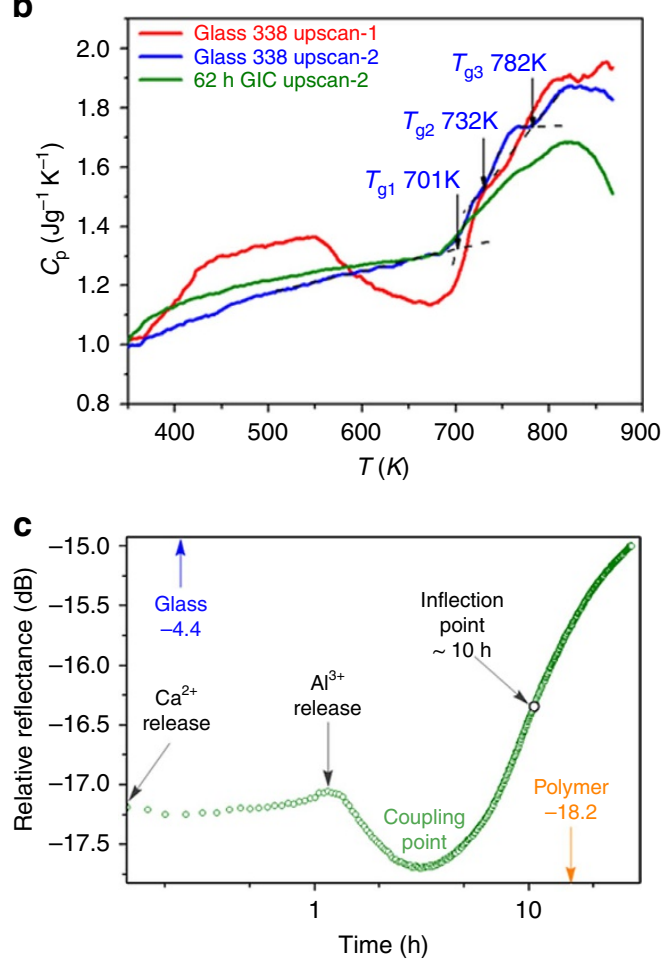

Figure 2 | GIC characterization. (a) TEM image of individual glass particle (above, scale bar, $100 \mathrm{~nm}$ ) with three distinguishable glass phases: GP1, GP2 and GP3 seen on an expansion of the white frame area (below, scale bar, $20 \mathrm{~nm}$, see text for details). (b) Three DSC upscan curves for the fresh G338 glass (red), the G338 glass subjected to the first up- and downscans (blue), and the GIC sample (green) subjected to $62 \mathrm{~h}$ setting and the subsequent up- and downscans, respectively. The red curve exhibits a water-loss endothermic response, followed by an exothermic enthalpy-release response; the blue upscan-2 curve reveals three sharp glass transitions $T_{\mathrm{g} 1}, T_{\mathrm{g} 2}$ and $T_{\mathrm{g} 3}$, which we associate with the glass phases GP1, GP2 and GP3, respectively; the green curve reveals how glass transitions are modified by setting. Both up- and downscan rates are $20 \mathrm{~K} \mathrm{~min}^{-1}$. (c) Coherent terahertz spectroscopy: changing sub-THz relative reflectance during setting, differentiating gel formation $\left(\mathrm{Ca}^{+2}\right.$ and $\mathrm{Al}^{3+}$ release) from chelation $\left(\mathrm{Al}{ }^{+3}\right.$ release); minimum $(\mathrm{CP})$ identifies the point where glass and polymer couple dynamically. 
a

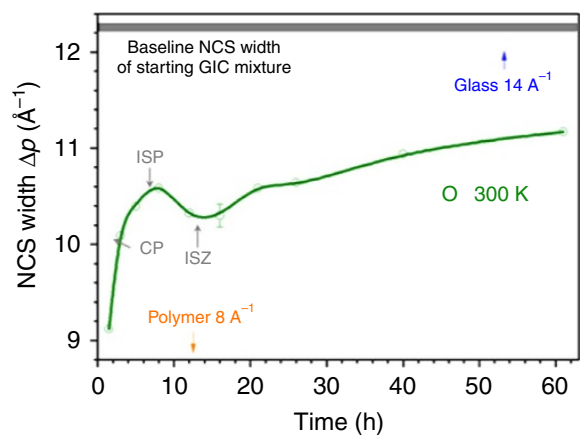

C
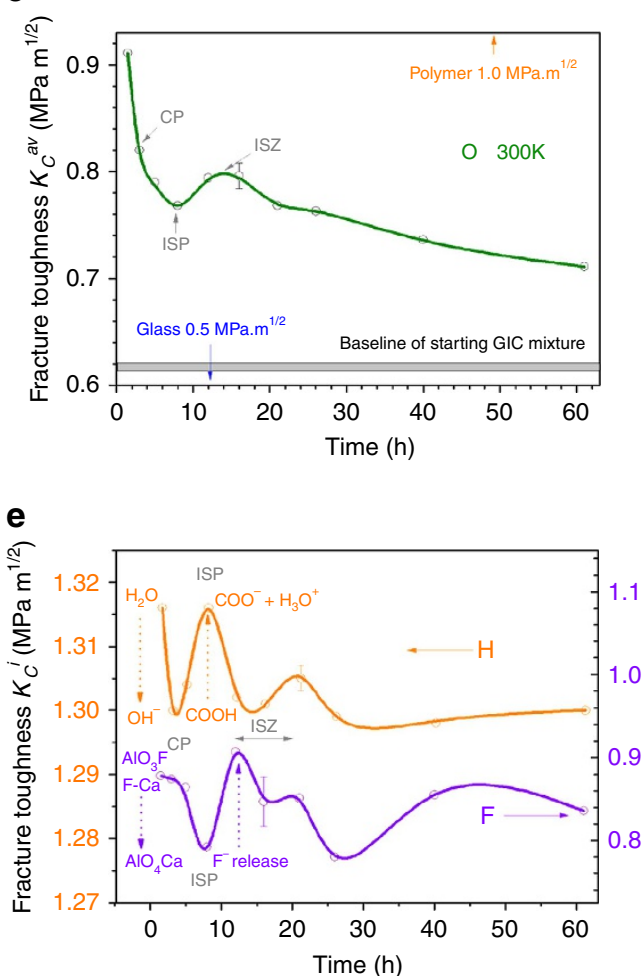

b

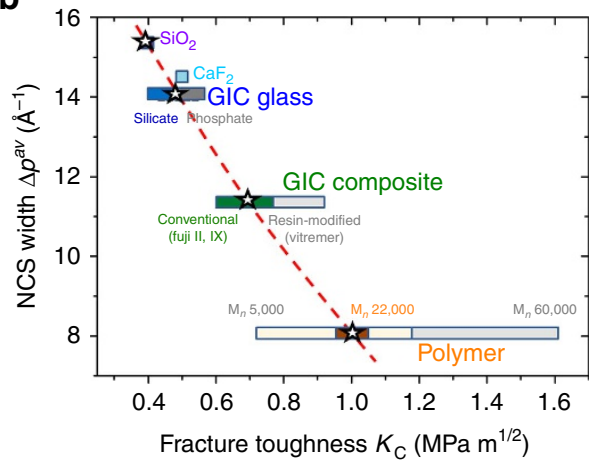

d

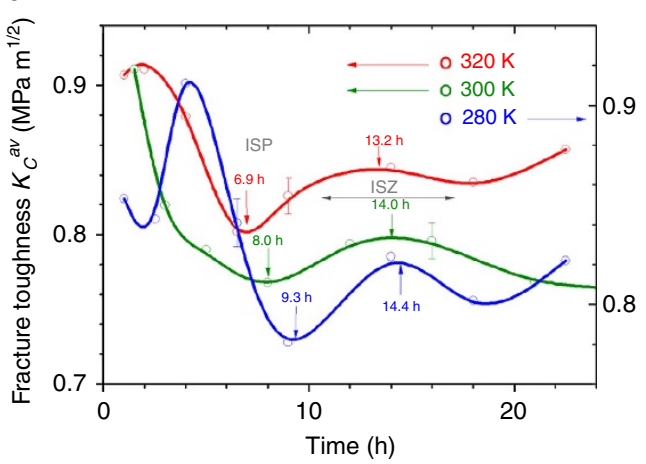

f

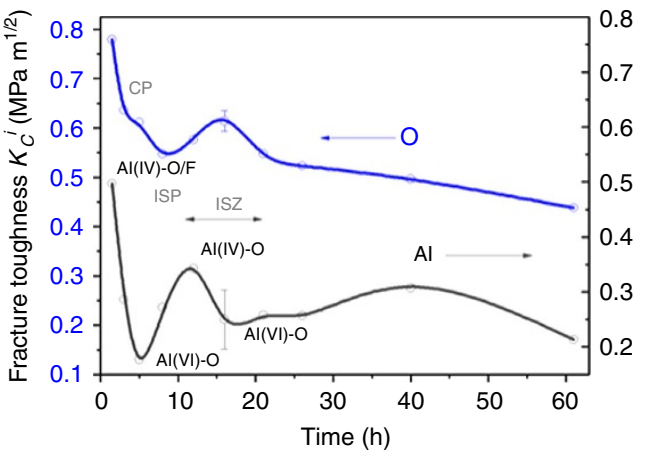

Figure 3 | Non-monotonic advancement in atom cohesion and fracture toughness during GIC solidification. (a) Overall GIC NCS peak width $\Delta p^{\text {av }}$ variations with setting time, measuring different stages in atomic cohesion during setting: CP, ISP and ISZ (see text for details). (b) Inverse relationship between atomic cohesion $\Delta p^{a v}$ and fracture toughness $K_{C}^{a v}$ of GIC, polymer and glass (asterisks) and associated materials (see text for details).

(c) Non-monotonic fall in overall $K_{C}^{\mathrm{av}}$ with setting time at $300 \mathrm{~K}$ obtained from overall $\Delta p^{\mathrm{av}} \mathbf{a}$ using $\mathbf{b}$, identifying CP and reaction points ISP and ISZ. (d) $K_{C}^{\mathrm{av}}(280 \mathrm{~K}), K_{C}^{\mathrm{av}}(300 \mathrm{~K})$ and $K_{C}^{\mathrm{av}}(320 \mathrm{~K})$, showing shifts in ISP and ISZ with setting temperature. (e) Fluctuations in $K_{C}^{i}$ for $\mathrm{H}$ and $\mathrm{F}$, showing evidence for hydration and fluorination. (f) $K_{C}^{i}$ for $\mathrm{O}$ and $\mathrm{Al}$ through the various setting stages. Elemental fracture toughness $K_{\mathrm{C}}^{i}$ were derived from $\Delta p_{\mathrm{i}}$ values (Supplementary Fig. 3a), with $K_{C}^{\mathrm{av}}(280,300$ and $320 \mathrm{~K})$ obtained from $\Delta p^{\mathrm{av}}(280,300$ and $320 \mathrm{~K}$ ) values (Supplementary Fig. 3b), in each case using b.

Following $62 \mathrm{~h}$ of setting, the $C_{\mathrm{p}}$ of the GIC (green curve) demonstrates the evolution of the glass-transition regions of the remaining glass.

Coherent terahertz spectroscopy (CTS $)^{33}$ has been employed to track changes in inter-particle binding at the interfaces through variations in collective low-frequency atomic dynamics during setting. Non-monotonic behaviour is clearly evident, revealing several large swings in the magnitude of $\mathrm{THz}$ dynamics (Fig. 2c).

For observing mechanical toughness $K_{\mathrm{C}}$ atomically, we have turned to in situ neutron Compton scattering (NCS) ${ }^{34}$, where neutron momentum recoil $\Delta p$ measures the atomic cohesion. The development of $\Delta p$ during setting at $300 \mathrm{~K}$ is clearly oscillatory (Fig. 3a; Supplementary Fig. 2). Using a new empirical relationship between the momentum recoil $\Delta p$ values and published fracture toughness values (Fig. $3 \mathrm{~b}$ ), changes in atomic
$K_{\mathrm{C}}$ have been analysed both for the total system $K_{\mathrm{C}}^{\mathrm{av}}$ (Fig. 3c,d) and for separate elements $K_{\mathrm{C}}^{\mathrm{H}, \mathrm{F}, \mathrm{O}, \mathrm{Al}}$ (Fig. 3e,f).

In situ neutron scattering measurements also reveal complementary variations in the structure factor $S(Q)^{35}$ associated with nanoscopic structure changes taking place during setting (Fig. 4a,c), and in the real-space transform $G(r)$ (Fig. 4b,d,e). This extensive set of in situ experiments has been used to quantify mechanical, structural and dynamical parameters during the setting of GICs, previously unobtainable atomistically ${ }^{7}$.

The G338 glass is chemically complex, containing ingredients for cementation (calcium, phosphate and alumino-silicate), mineralization (phosphate and fluoride), dental caries resistance (fluoride) and opal appearance ( $\mathrm{Ca}+\mathrm{F}$-rich particles). The network-forming ions are $\mathrm{Si}^{4+}, \mathrm{P}^{5+}$, with the majority being $\mathrm{Al}^{3+}$. $\mathrm{Al}^{3+}$ can be charge-compensated for tetrahedral 

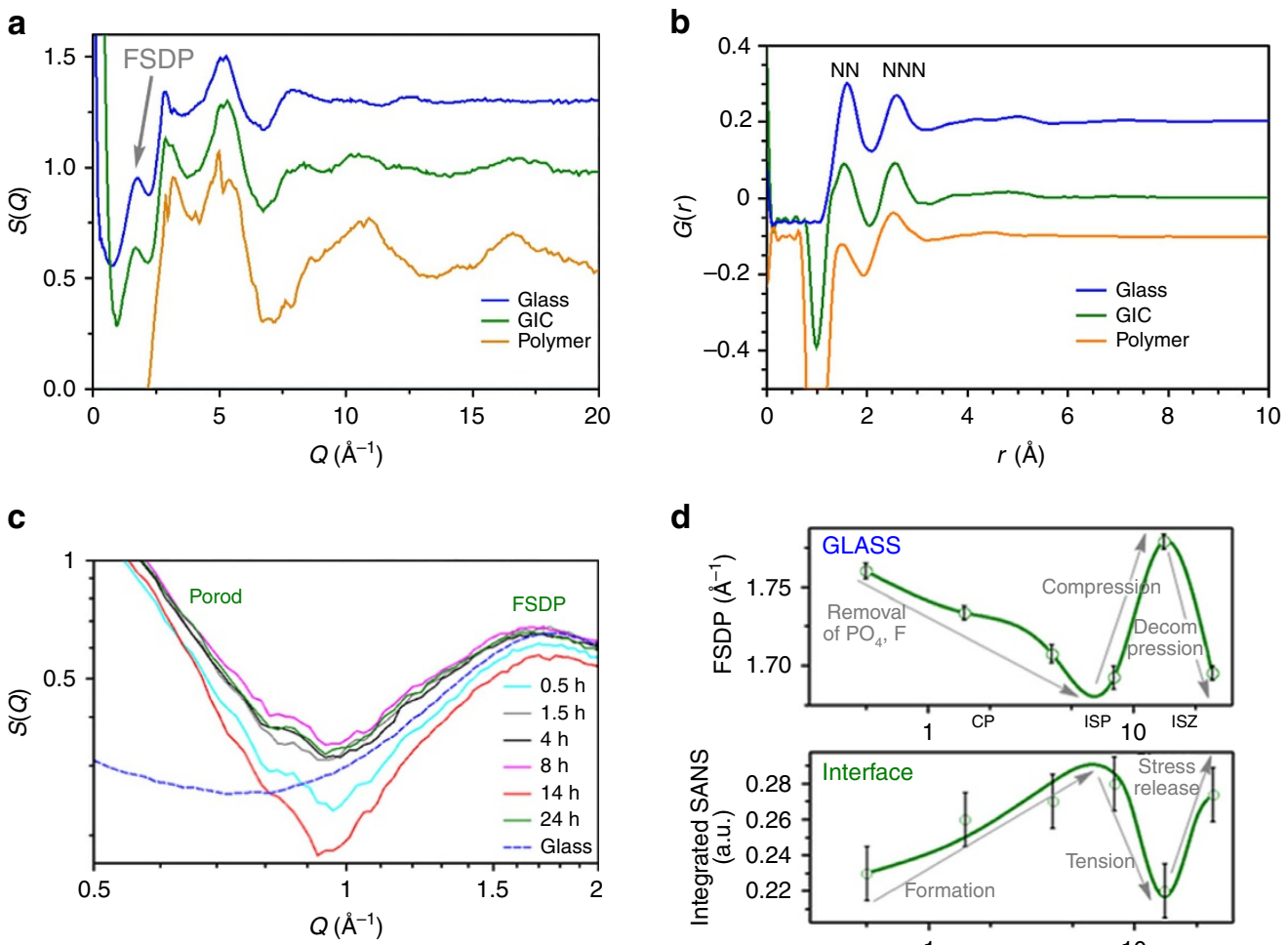

d
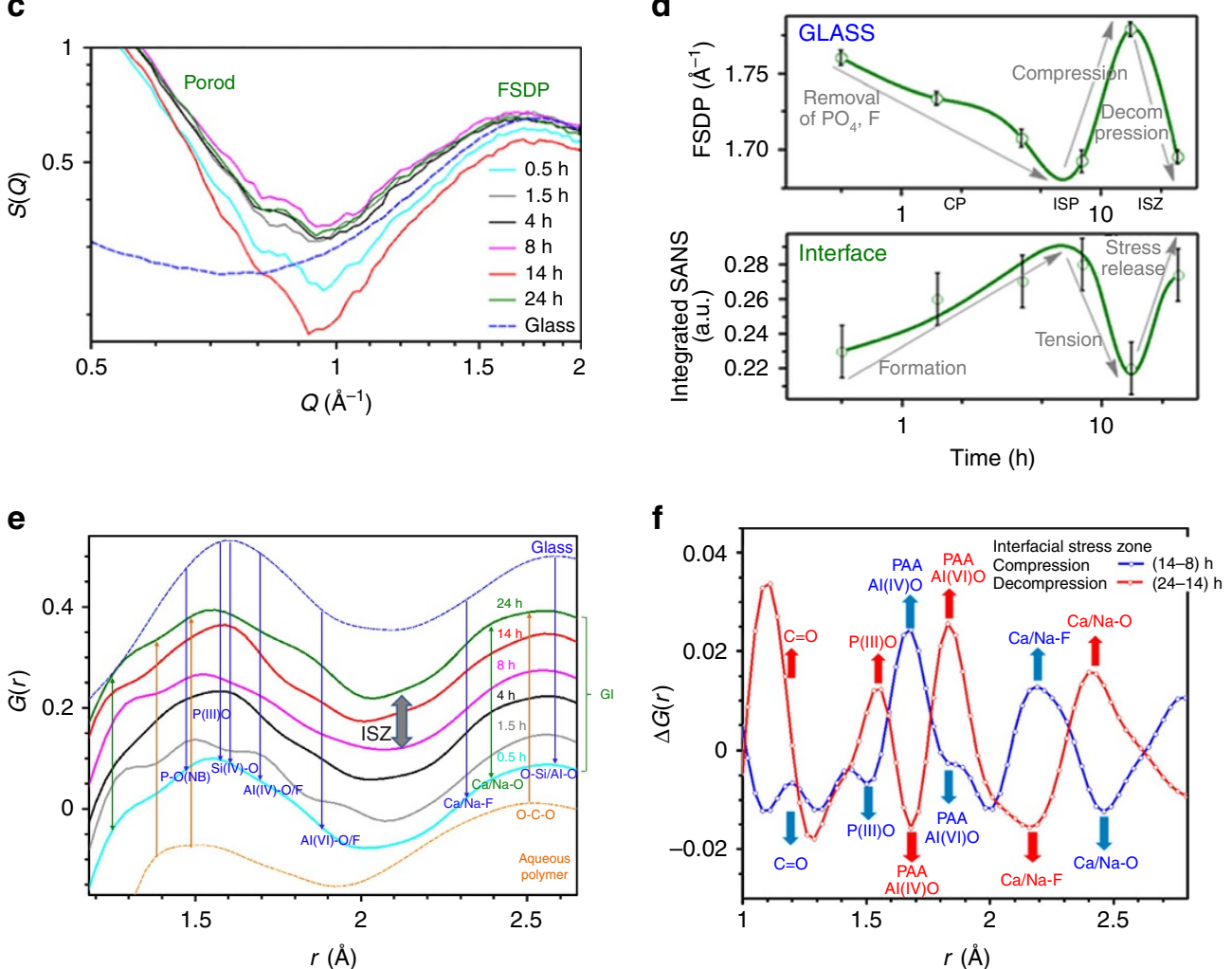

Figure 4 | Neutron scattering measurements. Time-averaged (a) $S(Q)$ and (b) $G(r)$, including FSDP and expected locations for nearest- and next-nearestneighbour pair correlations NN and NNN, respectively. (c) In situ time-resolved S(Q) over $24 \mathrm{~h}$ revealing changes in small-angle Porod scattering (SANS) at interfaces, and in the position of FSDP for the glass. (d) Fluctuations with setting in the FSDP position and the integrated SANS from c (Methods). Abrupt changes around $15 \mathrm{~h}$ coincide with the $\Delta p^{\text {av }}$ minimum and $K_{C}^{\text {av }}$ maximum (Fig. $4 \mathrm{a}, \mathrm{c}$ ). (e) In situ time-resolved $G(r)$ with NN distances for GIC related to glass (upper) and aqueous polymer (lower). (f) Trends in $\Delta G(r)$ obtained by differencing $G(r)$ across the ISZ (from $\mathbf{d}$ ) - splines for guiding the eye. $\mathbf{a}, \mathbf{b}$ and $\mathbf{e}$ data are all offset vertically for clarity of presentation.

configuration by $\mathrm{P}^{5+}$, and also by $\mathrm{Na}^{+}$and $\mathrm{Ca}^{2+}$, which, as network-modifiers, can promote the formation of non-BOs within the $\mathrm{BO}$ alumina-silicate network ${ }^{4,36}$. Considerable fluorine content further depolymerizes the glass melt, leading to low liquidus temperatures ${ }^{28}$. In the glass, $\mathrm{Al}^{3}+$ coordinates both to BOs and $\mathrm{F}^{-}$(ref. 36), while $\mathrm{F}^{-}$complexes with $\mathrm{Ca}^{2+}$ and $\mathrm{Na}^{+}$ as well as with $\mathrm{Al}^{3}+. \mathrm{SiO}_{4}$ and $\mathrm{PO}_{4}$ tetrahedra principally link via $\mathrm{BOs}$ to $\mathrm{Al}$ polyhedra, and, while these are mainly tetrahedral, $\mathrm{Al}(\mathrm{V})$ and $\mathrm{Al}(\mathrm{VI})$ configurations also occur, particularly at the developing interfaces, with the proportions changing during cement setting 28,36 .

The as-received G338 glass powder (Methods) comprises micron-sized particles (Fig. 2a) exhibiting extensive amorphous phase separation, as others have reported ${ }^{17,37}$. Our highresolution TEM image includes a continuous matrix $\left(\mathrm{GP}_{1}\right)$, in which are embedded $30-50-\mathrm{nm}$ spherical rosette domains $\left(\mathrm{GP}_{2}\right)$, decorated by $5-10-\mathrm{nm}$ droplets $\left(\mathrm{GP}_{3}\right) . \mathrm{GP}_{2}$ and $\mathrm{GP}_{3}$ are highlighted by large and small dashed circles, respectively, and are generally seen throughout this image and those of other particles. All three GPs are neutron amorphous (Fig. 4a) and the three glassy states can be verified by DSC traces (Fig. 2b). These reveal three glass transitions: $T_{\mathrm{g} 1}(701 \mathrm{~K}), T_{\mathrm{g} 2}(732 \mathrm{~K})$ and $T_{\mathrm{g} 3}$ $(782 \mathrm{~K})$. The small size of GPs and overlaying within TEM images prevents measurement of phase compositions to correlate with $T_{\mathrm{g}} \mathrm{s}$. However, as amorphous phase separation is the primary source for bulk crystalline nucleation in glass ceramics ${ }^{38}$, crystallographic studies of dental ceramics and devitrified GIC glasses ${ }^{37}$ identify possible glass-phase compositions. In particular, G338 glass, which is a typical phosphate-containing fluoroalumino-silicate glass, exhibits amorphous phase separation 
resembling the morphology in Fig. $2 \mathrm{a}$ and at least two glass transitions at similar temperatures to those appearing in Fig. $2 b^{37}$. Further annealing drives crystallization by bulk nucleation to a fluoro-phosphate phase and an alumino-silicate phase, the former emanating from amorphous phase-separated droplets and the latter from the surrounding matrix ${ }^{37,39}$. Accordingly, we attribute the surrounding matrix seen in our TEM images to an alumino-silicate GP (GP1 $T_{\mathrm{g} 1}$ ) and the nanophase droplets to a Ca-F-P-rich GP (GP3 $T_{\mathrm{g} 3}$ ). From the glass composition (see Materials in the Methods section) the remaining 30-50-nm spherical domains suggest a Ca-F-rich GP (GP2 $\left.T_{\mathrm{g} 2}\right)$. These attributions to the phase-separated components have been confirmed in a recent parallel study ${ }^{40}$.

Thermodynamically, the as-received G338 glass is far from equilibrium, as evidenced by the broad exothermic peak around $720 \mathrm{~K}$ that precedes the glass transitions (Fig. $2 \mathrm{~b}$ red curve). This shows the release of the enthalpy trapped during the very rapid quenching of the G338 melt ${ }^{41}$ and is absent on reheating (Fig. 2b blue curve). Accordingly, we expect each of the three separated amorphous phases $\mathrm{GP}_{1}, \mathrm{GP}_{2}$ and $\mathrm{GP}_{3}$ (Fig. 2a) to be structurally and energetically heterogeneous in the as-received glass, as discovered in other hyperquenched glasses ${ }^{41}$. Unstable phases will help drive the hydration process when PAA and glass are mixed.

By heating the GIC cement above room temperature, DSC reveals, first the release of water and any organic impurities and then the decomposition of the polymer, which coincides with the three $T_{\mathrm{g}} \mathrm{s}$ in the annealed glass (Supplementary Fig. 1a). The physical consequences of GIC setting are reflected in the altered glass-transition pattern of the residual glass (Fig. $2 \mathrm{~b}$ green curve) compared with the annealed G388 glass (Fig. 2b blue curve). Most obviously, the boundaries between all three glass transitions in the GIC cement are less distinct, suggesting that all the phases have reacted with PAA. This is most pronounced for GP2 $T_{\mathrm{g} 2}$ and GP3 $T_{\mathrm{g} 3}$, where the jump from glass to liquid is reduced, which will be partly due to their large surface area. Reactions with PAA might promote a disorder-order transition ${ }^{41}$, or even partial mineralization, both of which would lower the glass-transition peaks. By contrast, the onset temperature of GP1 $T_{\mathrm{g} 1}$ is least affected by setting, indicating that the primary alumino-silicate network remains largely intact during setting.

Cooperative interfacial dynamics. We observe time-dependent changes in the sub-THz range using $\mathrm{CTS}^{33}$ in the early stages as GIC cementation advances (Fig. 2c). These occur between the separate CTS values of the glass and polymer. Since bulk values will not vary, the changes that we see must relate directly to the low-frequency dynamics developing at the interfaces between glass and polymer, as well as those between crosslinked polymer chains within the matrix during setting. The vibrational modes in the sub- $\mathrm{THz}$ energy are centred around $0.5 \mathrm{THz}$ and mainly involve collective motions of constituent atoms. These will be increasingly added to, during setting, by inter-component librational changes such as twisting, bending and flexing, with interfacial links serving as pivot points. As these encompassing motions modulate macroscopic interfacial and mechanical properties $^{29,30,32}$, such as plasticity and elasticity ${ }^{31}$, the initial dip in CTS signal coincides with $\mathrm{Ca}^{2+}$ release from the glass. This would appear to be due to the cationic effusion. Governed by the $\mathrm{Ca}^{2+}$ rattling frequency in the glass $(\sim 12 \mathrm{THz})^{42}$, this would initially outpace the polymer's ability to deform rapidly enough to bind the excess ions, being limited by the polymer's intrinsic low-frequency dynamics $(\sim 0.5 \mathrm{THz})$. Once $\mathrm{Ca}^{2+}$ is released from the glass, however, subsequent signal recovery over the first $1 \mathrm{~h}$ traces the polymer's progressive chelation of $\mathrm{Ca}^{2+}$ at the interface, until $\mathrm{Al}^{3+}$ emerges from the glass network at $\sim 1.5 \mathrm{~h}$, initiated by a sharp drop in reflectance continuing to $\sim 5 \mathrm{~h}$. As the polymer is increasingly localized into a percolating matrix around the glass powder ${ }^{13}$ and, with gel formation on the glass-particle surfaces ${ }^{4,5}$, we would expect an associated dampening of the CTS signal, approaching the values of the isolated polymer component (Fig. 2c). This drop, however, is then followed by a sharp increase in $\mathrm{THz}$ reflectance, signalling increased activation of interfacial collective modes, and thus of increased coupling between polymer and alumino-silicate glass components. Accordingly, the minimum at $3 \mathrm{~h}$, we propose, defines a coupling point $(\mathrm{CP})$ in the reaction-setting mechanism, after the cement has lost its initial plasticity (Fig. 1d), but before it has started to establish its mechanical strength. More generally, modulation of fluctuations in the sub-THz-regime generally has been linked to elasticity and shear-induced phase transitions ${ }^{43}$ and has also been associated with the stability of zeolite structures $^{44}$, proteins ${ }^{32}$ and in general is typical of the librational dynamics of two-level systems in network structures ${ }^{42}$.

Atomic cohesion and fracture toughness changes. The momentum peak widths $\Delta p_{\mathrm{i}}$ of individual atom types $i$, measured by NCS (Supplementary Fig. 3), quantify the depths of interatomic potentials, and these relate directly to atomic cohesion $^{34}$. Marked oscillatory changes in $\Delta p_{\mathrm{i}}$ occur during GIC setting (Supplementary Fig. 3a), particularly over the first day. To obtain the average momentum width $\Delta p^{\text {av }}$ representative of all elements in the setting cement, $\Delta p_{\mathrm{i}}$ are combined as $\Sigma_{i} c_{i} \Delta p_{i}$, where $c_{\mathrm{i}}$ is the element fraction. Note that $\Delta p^{\mathrm{av}}$ is bounded by $\Delta p^{\text {polymer }}$ and $\Delta p^{\text {glass }}$ widths for respective polymer and glass components, measured separately (Fig. 3a). As setting advances, $\Delta p^{\text {av }}$ starts close to the $\Delta p^{\text {polymer }}$ width, and increases over $62 \mathrm{~h}$, levelling off below the width of the starting mixture, which is dominated by glass $\Delta p^{\text {glass }}$. During this time there is an inflection point at $\sim 5 \mathrm{~h}$, which we have identified from CTS $\mathrm{THz}$ spectra as the CP between glass and polymer. This is followed by a clear maximum at $\sim 8 \mathrm{~h}$, where the atomic cohesion is greatest. We define this as the initial setting point (ISP). This might be the desirable point for cementation to halt. However, there is then a minimum at $\sim 15 \mathrm{~h}$, where atomic cohesion momentarily drops before recovering. We later identify this, from changes in $S(Q)$ (Fig. 4d), as an interfacial stress zone (ISZ). Thereafter, the average momentum width $\Delta p^{\text {av }}$ starts to level out.

Since NCS probes atomic cohesion ${ }^{34}$, when $\Delta p^{\text {av }}$ is lower, average atomic cohesion is also lower, interatomic potentials shallower and wider and thus the material is tougher, and vice versa. Accordingly, we expect that $\Delta p^{\mathrm{av}}$ and fracture toughness $K_{\mathrm{C}}$ might be inversely related for groups of materials. This is demonstrated in Fig. $3 \mathrm{~b}$ for the GIC system studied, where $\Delta p^{\text {av }}$ values for this GIC composite measured at $24 \mathrm{~h}$ are plotted, together with $\Delta p^{\text {polymer }}, \Delta p^{\text {glass }}$ measured separately, as well as those of related compounds (see Supplementary Materials). All are plotted directly as a function of values of fracture toughness $K_{\mathrm{C}}$ reported in the literature ${ }^{21,45-47}$. These extend from single values for specific materials, such as $\mathrm{SiO}_{2}$ or $\mathrm{CaF}_{2}$, to ranges of values of $K_{\mathrm{C}}$ for different systems such as glasses and polymers, which span different compositions and material treatments. The asterisked values relate to the particular components, compositions and preparation protocol used in this study (Methods). The spread of the $K_{\mathrm{C}}$ values around these asterisked points for other glasses, GICs and polymer systems extend to smaller or larger values. These are smallest for oxide glasses and largest for phosphate glasses. Fracture toughness of the GIC glass falls midrange defining the value used in Fig. 3b. Compared with glasses, the span of $K_{\mathrm{C}}$ is much larger for polymers, where $K_{\mathrm{C}}$ is strongly governed by molecular weight $M_{\mathrm{n}}$ (ref. 45), which, for 
the polymer used for the present GIC, lies close to 22,000 (refs 7,9) and determines the asterisked value in Fig. 3b. The value of $M_{\mathrm{n}}$ in turn influences the mechanical properties not just of polymers but also of $\mathrm{GICs}^{48,49}$. In particular, $K_{\mathrm{C}}$ is greater for resin-modified GICs than for conventional GICs where the current GIC system falls midway, which determines the final asterisked value. Taken together, these well-defined points result in our empirical relationship between $\Delta p^{\text {av }}$ and $K_{\mathrm{C}}$ being also well-defined. This is not a reciprocal relationship, as the negative slope, $\mathrm{d} \Delta p^{\mathrm{av}} / \mathrm{d} K_{\mathrm{C}}$, decreases with increasing $K_{\mathrm{C}}$. Figure $3 \mathrm{~b}$ provides a practical look-up table to calibrate $\Delta p^{\text {av }}$ widths in $\AA^{-1}$ measured with NCS with fracture toughness in MPa ${ }^{1 / 2}$, and is used to convert $\Delta p^{\text {av }}$ from Fig. 3a into average fracture toughness values $K_{C}^{\mathrm{av}}$ at $300 \mathrm{~K}$ during setting (Fig. 3c).

Both atomic cohesion measured from $\Delta p^{\text {av }}$ using NCS and fracture toughness $K_{\mathrm{C}}^{\mathrm{av}}$ are opposite sides of the same coin and reveal the same CP, ISP and ISZ features in monotonic setting observed early on (Fig. $3 \mathrm{a}, \mathrm{c})$. Moreover, $K_{\mathrm{C}}^{\mathrm{av}}$ obtained from NCS observations offers a way to continuously access mechanical toughness in situ, otherwise complicated by the statistical averaging of multiple specimens inherent in retrospective destructive fracture testing ${ }^{18}$. In particular, $K_{\mathrm{C}}^{\mathrm{av}}$ drops sharply with setting time (Fig. $3 \mathrm{c}$ ), in line with the anticipated fall in $v$ (Fig. 1d), followed by the coupling (CP), setting (ISP) and stress (ISZ) points. Thereafter, $K_{\mathrm{C}}$ levels out over the next 2 days, significantly above the baseline toughness-derived using the mixing ratio of the glass and polymer components' $K_{\mathrm{C}}$ values (Fig. 3c). We observe similar progression in $K_{C}^{\mathrm{av}}$ at different setting temperatures over the first day (Fig. 3d; Supplementary Fig. 3b). These reveal expected upward shifts in temperature with time for the features CP, ISP and ISZ, as the setting temperature increases.

The elemental momentum widths $\Delta p_{i}$ (Supplementary Fig. 3a) analysed from the single-particle momentum distribution (Supplementary Fig. 2b) can be converted into elemental fracture toughness values $K_{\mathrm{C}}^{\mathrm{i}}$ using the same empirical relationship (Fig. 3b). These are shown for $\mathrm{H}, \mathrm{F}, \mathrm{O}$ and $\mathrm{Al}$ (Fig. 3e,f) and share the same overall scale as $K_{\mathrm{C}}^{\text {av }}$ (Fig. $3 \mathrm{c}$ ), ranging from $\mathrm{H}$ with the highest fracture toughness values $\left(\sim 1.3 \mathrm{MPa} \mathrm{m}^{1 / 2}\right)$ to $\mathrm{Al}$ with the lowest $\left(\sim 0.3 \mathrm{MPa} \mathrm{m}^{1 / 2}\right)$. Elemental values can be interpreted as imparting different degrees of fracture toughness at the atomistic level, with $\mathrm{H}$ and $\mathrm{Al}$, for example, possibly contributing toughness and brittleness, respectively. The reaction points $\mathrm{CP}$, ISP and ISZ are again evident. However, $\mathrm{H}, \mathrm{F}$ and $\mathrm{Al}$ exhibit oscillatory changes in $K_{\mathrm{C}}^{\mathrm{i}}$ (and $\Delta p_{\mathrm{i}}$ ) with setting time (Fig. 3e,f; Supplementary Fig. 3a), which are averaged out in $K_{\mathrm{C}}^{\mathrm{av}}$. These may be associated with developments in hydration $\left(K_{\mathrm{C}}^{\mathrm{H}}\right)$, fluorination $\left(K_{\mathrm{C}}^{\mathrm{F}}\right)$ and chelation $\left(K_{\mathrm{C}}^{\mathrm{Al}}\right)$, respectively. This sensitivity to differing chemical bonding is illustrated by hydrogen (Supplementary Fig. 3a). Eventual $\Delta p^{\mathrm{H}}$ for GICs reported here is $4.81 \AA^{-1}$, similar to water $\left(4.84 \AA^{-1}\right)$, but greater than $\mathrm{ZrH}_{2}\left(4.15 \AA^{-1}\right)$ or $\mathrm{NaH}\left(3.32 \AA^{-1}\right)^{34,50,51}$.

We consider that variable $\mathrm{H}$ cohesion during setting has two principal staggered sources: (A) hydration of the alumino-silicate GP GP1 $\left(\mathrm{H}_{2} \mathrm{O}+\equiv \mathrm{Si} / \mathrm{Al}-\mathrm{O}-\mathrm{Si} / \mathrm{Al} \equiv \rightarrow \equiv \mathrm{Si} / \mathrm{Al}-\mathrm{OH}^{-}+-\mathrm{OH}-\mathrm{Si} /\right.$ $\mathrm{Al} \equiv)$ binding $\mathrm{OH}^{-}$; (B) PAA carboxylation at the interfaces $\left(\mathrm{COOH} \leftrightarrow \mathrm{COO}^{-}+\mathrm{H}^{+}\right)$freeing $\mathrm{H}^{+}$or $\mathrm{H}_{3} \mathrm{O}^{+}$. (A) is likely to be linked with gelation at $\mathrm{CP}$, and (B) with subsequent chelation of $\mathrm{Al}$ at the ISP that follows at the interfaces. This is consistent with the first minima (binding of $\mathrm{OH}^{-}$) and maxima (release of $\mathrm{H}_{3} \mathrm{O}^{+}$) in $K_{\mathrm{C}}^{\mathrm{H}}$ with setting time (Fig. 3e). Likewise, the subsequent maximum in $K_{\mathrm{C}}^{\mathrm{F}}$ corresponds to low atomic cohesion, which can be associated with release of $\mathrm{F}^{-}$. The considerable quantity of $\mathrm{F}^{-}$in the glass substantiates reports of $\mathrm{AlO}_{3} \mathrm{~F}$ centres charge balanced by F-Ca and F-Na (ref. 36), which in G338 probably originate from both GP2 and GP3 GPs (Fig. 2a).
Accordingly, we interpret oscillatory changes in $K_{\mathrm{C}}^{\mathrm{F}}$ with setting time principally with the transformation of oxyfluorides into oxides at the interfaces, accompanied by the release of $\mathrm{F}^{-}$when the atomic cohesion is least (Fig. 3e). Indeed, the similarity of $K_{\mathrm{C}}^{\mathrm{F}}$ to $K_{\mathrm{C}}^{\mathrm{Al}}$ (Fig. $3 \mathrm{e}, \mathrm{f}$ ) points to $\mathrm{F}^{-}$and $\mathrm{Al}^{3+}$ being partners in the same process, $\mathrm{AlO}_{3} \mathrm{~F}$ converting to $\mathrm{AlO}_{4}$ at the ISP. The leaching of $\mathrm{F}^{-}$during early setting provides caries resistance while actively stimulating tooth tissue remineralization, as is often claimed in the literature 711,37 . It seems likely, too, that the compositional species from the Ca, F+P-rich GP (GP3), which assists tooth mineralization ${ }^{10}$, will be released during the corrosion of the glass at CP. Finally, because oxygen is involved in hydration, fluorination and chelation, we expect setting variations in $K_{\mathrm{C}}^{\mathrm{O}}$ will closely follow the overall fracture toughness $K_{C}^{\mathrm{av}}$, which indeed appears to be the case, including the ISZ maximum close to $\sim 15$ h (Fig. 3c,f).

Atomic structure and interfacial stress. Time-averaged structure factors $S(Q)$ (where $Q$ is the neutron scattering vector) and total radial distribution functions $G(r)$ (where $r$ is the interatomic distance, with nearest-neighbour (NN) and next $\mathrm{NN}(\mathrm{NNN})$ ) for the GIC, glass and polymer components measured separately are compared in Fig. 4a,b. $S(Q)$ for the GIC and polymer include artefacts in amplitude, relating to the featureless, but substantial, incoherent scattering of hydrogen (Methods). The peak positions on $Q$ axis in $S(Q)$ and in $G(r)$, though are reliable. Deuteration was avoided in this study, because this would have adversely affected diffusion and cement-setting processes.

Both GIC and glass $S(Q)$ s exhibit an initial peak-known as the first sharp diffraction peak (FSDP). This directly reflects the average length of intermediate-range order domain $r_{\text {IRO }}$ in oxide glasses ${ }^{42}$. The position of the FSDP $Q_{\mathrm{FSDP}}\left(2 \pi / r_{\mathrm{IRO}}\right)$ is composition- and pressure dependent. The fluctuating position of the FSDP (Fig. 4c,d) therefore indicates changes in composition and internal stress. Q Q FDDP for G338 glass is located between $1.5 \AA^{-1}$ for silica and alumina-silicate glasses and $1.9 \AA^{-1}$ for phosphate glasses ${ }^{42}$. We therefore attribute the initial decrease in $Q_{\text {FSDP }}$ for GIC (Fig. 4d) to the release of phosphate from the Ca, $\mathrm{F}+\mathrm{P}$-rich GP (GP3 Fig. 2a). Beyond the ISP there is a sharp increase consistent with compression developing in the remaining glass, followed by decompression, defining the start and end of ISZ. The internal pressure in the glass can be estimated by comparing the shift in $Q_{\mathrm{FSDP}}$ for silica with the permanent density change under pressure ${ }^{52}$, and points to $\sim 1 \mathrm{GPa}$ being generated in the glass after the ISP at $\sim 8 \mathrm{~h}$, then released by $\sim 24$ h (Fig. $4 \mathrm{~d}$ ).

The integrated small-angle neutron scattering (SANS) from 0.5 to $1 \AA^{-1}\left(\int_{Q_{\min }}^{Q_{\max }} S(Q) Q^{2} \mathrm{~d} Q\right)$ from Fig. $4 \mathrm{c}$ is also shown in Fig. 4d. SANS, which measures differential density at the nanolevel, is dominated here $\left(\sim 0.5 \AA^{-1}\right)$ by Porod scattering from interfaces ${ }^{42}$, which in this case will be principally between the polymer and glass components (Fig. 4c). As the major amorphous phase separation structure in the glass $\left(\mathrm{GP}_{2}\right)$ is $\sim 50 \mathrm{~nm}$ in size (Fig. 2a), equivalent to $\sim 0.002 \AA^{-1}$, it is out of range for the present SANS experiments. Instead SANS intensity, which is proportional to interfacial density within the GIC, increases towards the ISZ and then drops sharply before recovering at $\sim 24 \mathrm{~h}$; this indicates initial tension at the hybrid interfaces when the density is lowest, then stress release over the ISZ when the original density recovers (Fig. 4d).

Taken together, these variations in nanoscopic structure start with decreased density contrast pointing to tension at the interfaces (SANS) as the glass compresses (increase in FSDP $Q_{\mathrm{FSDP}}$ ) constrained by the increasingly rigid surrounding matrix. This is followed by overall stress release, where density contrast at 
the interfaces observed from SANS and pressure from the decrease in $Q_{F S D P}$ is restored, and suggests interfacial failure develops over the later stages of the ISZ. Indeed, indentation studies have shown that macroscopic cracks occur at GIC hybrid interfaces ${ }^{13}$. Potential failure should occur via $\mathrm{Al}^{3+}$ linkages, as an alternative to fracture propagation at ionic sites within the polysalt complex, which has also been suggested ${ }^{48}$, where $\mathrm{Al}^{3+}$ bonding may also play an important role.

To ascertain whether complementary changes in real-space structure occur, we have turned to time-dependent total radial distribution functions, $G(r)$ s (Fig. 4e). Because the hydrogen content of the polymer and GIC distorts amplitudes, conventional pair-distribution analysis was replaced by differencing out the effects of hydrogen in the setting of GIC by using $\Delta G(r) \mathrm{s}$ (Fig. $4 \mathrm{f}$ ). Furthermore, $\Delta G(r)$ s also masks the unreacted glass and polymer components, highlighting changes in local atomic structure at the interfacial regions during cementation.

Separately measured $G(r)$ s for GIC, glass and polymer exhibit two well-resolved peaks, due to $\mathrm{NN}$ and $\mathrm{NNN}$ interatomic distances, respectively (Fig. 4b). This complex multicomponent composite includes numerous interatomic distances within the $\mathrm{NN}$ and NNN envelopes, related both to the polymer and glass, as well as to the organic-inorganic interfaces. Figure $4 \mathrm{e}$ is therefore annotated with the principal atomic pair correlations in the polymer, such as $\mathrm{C}=\mathrm{O}$ and $\mathrm{C}-\mathrm{C}$, and $\mathrm{Al}-\mathrm{O}_{4}, \mathrm{Al}-\mathrm{O}_{6}$ and $\mathrm{O}-[\mathrm{Si} /$ $\mathrm{Al}]-\mathrm{O}$ in the G338 glass; as well as fluorinated environments identified spectroscopically ${ }^{36}$, including F-Ca and F-Na. None of these can be unequivocally identified.

Measurable changes occur in $G(r)$ with setting, however, enhanced with differencing, which effectively removes unreacted components in the bulk (Fig. 4e,f). Notably, the coordination of interfacial $\mathrm{Al}(\mathrm{IV}) \mathrm{O}$ transforms to $\mathrm{Al}(\mathrm{VI}) \mathrm{O}$ over the ISZ, with complementary fluctuations in the carboxylate $(\mathrm{C}=\mathrm{O})$ correlations, coinciding with generation and then release of internal interfacial stress (Fig. 4d,f). In addition, the switch between $\mathrm{Ca} / \mathrm{Na}-\mathrm{F}$ and $\mathrm{Ca} / \mathrm{Na}-\mathrm{O}$ is consistent with discharge of $\mathrm{F}$ (Fig. $3 \mathrm{e}$ ). Both swings in nanoscopic structure-SANS and FSDP (Fig. 4d) and in atomic structure $-\Delta G(r)$ (Fig. $4 \mathrm{f}$ ), coincide with the rise and fall of toughness $K_{\mathrm{C}}^{\mathrm{av}}$ around $15 \mathrm{~h}$ (Fig. 3c) and the complementary minimum in atomic cohesion (Fig. 3a).

\section{Discussion}

Comparatively few materials have been measured to date using NCS-GICs being the first complex and dynamic system to be studied. Necessarily, there are differences in NCS profiles with temperature, pressure and interatomic bonding, the latter having provided the avenue to track changing atomic cohesion and dynamic interfacial configurations during cementation. Comparisons between elemental NCS peak widths $\Delta p_{\text {i }}$ (Supplementary Fig. 3a) and overall $\Delta p$ (Supplementary Fig. $3 \mathrm{~b}$ ) and fracture toughness therefore need to relate to the specific materials studied-glass, GIC or polymer-and for them to be measured under the same conditions.

Such geometric switching, mainly dynamic configurations of $\mathrm{Al}$ ranging from four-coordinate tetrahedra originating in the glass to a five-coordinate pyramidal, is the likely initial GIC-setting sequence between CP and the ISP. The coherent $\mathrm{THz}$ frequencies detected at the developing hybrid interface are in the collective mode range, comprised of twisting, rocking, flexing and compressing modes-potentially at flexural Al pivots. Importantly, these fall among the collective modes reported for $\mathrm{SiO}_{2}$ (ref. 44), inorganic glasses and zeolite structures ${ }^{44}$, and typical of the dynamics of two-level systems ${ }^{42}$.

The successful juxtaposition of in situ sub-THz spectroscopy and neutron methods with DSC herein has enabled the complex setting-reaction processes of GIC dental composites to be unravelled structurally, energetically and dynamically at the atomic level for the first time. Our methods are equally applicable to studying fracture-toughness development in modified bioactive cement compositions ${ }^{53}$, where setting may be faster or slower, or in resin-modified GICs before and after curing, including the collective $\mathrm{THz}$ dynamics, which, in turn, are linked to interparticle and interfacial dynamics. We expect that this battery of techniques will also offer advantages more generally for studying mechanical toughness microscopically and non-destructively in other types of mercury-free cements during setting.

\section{Methods}

Materials. The composition of the G338 glass powder (First Scientific Dental $\mathrm{GmbH}$, Elmsohm, Germany) was $\mathrm{Na}_{6.3} \mathrm{Ca}_{6.6} \mathrm{P}_{6.2} \mathrm{Al}_{16.9} \mathrm{Si}_{11.8} \mathrm{O}_{32.5} \mathrm{~F}_{19.7}$ (ref. 7) and was hand-mixed with Chemflex liquid (Dentsply DeTrey GmbH, Germany) of $40 \%$ polyacrylic acid solution, in a respective 2.5:1 ratio for all experiments (Fig. 1b), the proportions leaving the aqueous polymer fraction above the percolation threshold (16\%), ensuring that glass particles were fully enveloped. Fresh cement was promptly loaded into measurement canisters. Setting time was recorded from the start of mixing. In each case, the powder and polymer were similarly measured separately, the 2.5:1 ratio providing the baseline $t=0$ values (Fig. 3a,c).

Transmission electron microscopy. TEM was performed at the NanoVision Centre at QMUL using scanning electron microscopy and TEM facilities. The G338 glass powder was dried before mounting. With an average particle size range of $\sim 4 \mu \mathrm{m}$ (Supplementary Fig. 4), many smaller fragments of $\sim 1 \mu \mathrm{m}$ were also imaged, revealing phase separation of $50-\mathrm{nm}$ rosette globules within a matrix (Fig. 2a)

Differential scanning calorimetry. The isobaric heat capacity $\left(C_{\mathrm{p}}\right)$ data for both G338 and the GIC sample subjected to the $62 \mathrm{~h}$ setting and subsequent first DSC up- and downscans (Fig. 2b) were collected in an argon atmosphere using a Netzsche STA449C; reproducibility being checked for baseline drift. G338 underwent two runs of up- and downscan (see glass 338 upscan-1 and upscan-2 curves). For scan 1, the sample was held for $5 \mathrm{~min}$ at $323 \mathrm{~K}$, heated at $20 \mathrm{Kmin}^{-1}$ to $873 \mathrm{~K}$ and then cooled back to $523 \mathrm{~K}_{\text {at }} 20 \mathrm{~K} \mathrm{~min}^{-1}$. Scan 2 followed the same procedure. $C_{\mathrm{p}}$ was determined using a sapphire reference.

Supplementary Fig. 1 shows two upscan curves for both the DSC output (isobaric heat capacity $C_{\mathrm{p}}$ ) and the mass change of the GIC sample, which was subjected to a 62 -h setting. The sample was upscanned in argon at $20 \mathrm{Kmin}^{-1}$. The first upscan curve in Supplementary Fig. 1a displays two endothermic responses indicating the water evaporation between 340 and $600 \mathrm{~K}$, and the PAA decomposition between 600 and $800 \mathrm{~K}$. These two thermal responses are reflected in the two-stage drop in mass as shown in Supplementary Fig. $1 \mathrm{~b}$.

Coherent terahertz spectroscopy. Spectra of G338, Chemflex and the setting cement (Fig. 2c) were obtained using a unique coherent $\mathrm{THz}$ transceiver ${ }^{33}$ incorporating vector-network-analyser-driven quasi-optical circuitry (Supplementary Fig. 5). The cement was loaded into a THz-transparent (polyethylene) vessel (Supplementary Fig. 6) and set at the focal point of fast mirrors, F2 and sealed to maintain water content. The sample absorbance was recorded as spectral reflectance response relative to a standard flat aluminium reflector.

Neutron Compton scattering. NCS spectra were obtained using VESUVIO spectrometer at the ISIS neutron source, with sample sizes of $55 \mathrm{~g}$ glass, $20 \mathrm{~g}$ polymer and $14 \mathrm{~g}$ cements ( $10 \mathrm{~g}$ glass $+4 \mathrm{~g}$ polymer-to give $2.5: 1 \mathrm{mix}$ ratios), and repeated at differing temperatures $(280,300$ and $320 \mathrm{~K})$. Under the conditions of high neutron energy transfer, $1-30 \mathrm{eV}$, and wavevector transfer, $30-200 \AA^{-1}$, the impulse approximation is valid ${ }^{34}$. This treats the neutron scattering event as involving a single atom, with conservation of the total kinetic energy and momentum of the neutron plus the atom. NCS therefore probes the momentum distribution $n_{\mathrm{i}}(p)$ of each element $i$ present in the sample.

Calibration was first done by measuring the empty beam and the empty sample holder. The empty sample holder spectrum and the multiple scattering were always subtracted before any data analyses. Multiple scattering was calculated using the assessed Monte Carlo code DINSMS ${ }^{54}$, which is routinely used for multiplescattering determinations for NCS experiments. In particular, the code calculates the time-of-flight spectrum of multiply scattered neutrons from samples of known geometry and compositions in DINS experiments. Such a contribution can be isolated in the simulation and subtracted from the experimental data; a detailed description can be found in ref. 54. The raw time-of-flight data for each singledetector spectrum were fitted using the known stoichiometry of the sample. The fitting was carried out after correction for multiple scattering and subtraction of the sample canister signal. Examples are shown in Supplementary Fig. 2 for forward 
and backward geometries. These include fitting to NCS momentum widths $\Delta p_{\mathrm{i}}$ for GIC elements present as shown, taking advantage of the instrument's ability to probe single-particle momentum distribution ${ }^{55,56}$. The individual element-specific time-of-flight data were then transferred to momentum space, and then the peak width of each element $\Delta p_{\mathrm{i}}$ was calculated in $\AA^{-1}$ as full width at half maximum (Supplementary Fig. 3), following existing procedures ${ }^{34}$. Also included is the difference between adjacent time slices close to the ISZ (Fig. 3c). The settingdependent elemental momentum widths $\Delta p^{\mathrm{H}}, \Delta p^{\mathrm{F}}, \Delta p^{\mathrm{O}}$ and $\Delta p^{\mathrm{Al}}$ used to create the mechanical fracture toughness plots in Fig. 4e,f are shown in Supplementary Fig. 3a and exhibit significant variations with setting time. The overall average momentum widths $\left(\Sigma_{i} c_{i} \Delta p_{i}\right.$ where $c_{i}$ is the elemental atomic fraction) for setting at 280,300 and $320 \mathrm{~K}, \Delta p^{280 \mathrm{~K}}, \Delta p^{300 \mathrm{~K}}, \Delta p^{320 \mathrm{~K}}$, from which $K_{\mathrm{C}}^{\text {av }}$ plots in Fig. $3 \mathrm{~d}$ were obtained, are plotted in Supplementary Fig. $3 \mathrm{~b}$.

Neutron diffraction. Data were collected using NIMROD at ISIS ${ }^{35}$. Calibration was done using a vanadium reference, after which glass powder and liquid were measured in a TiZr cell. Setting GIC measurements were performed over $24 \mathrm{~h}$ Data were analysed using the Gudrun program package ${ }^{57}$ to correct for the contributions from the empty cell, instrument background and to normalize the data to absolute units using the scattering of a vanadium standard absorption before attenuation and multiple-scattering corrections. Correction for the contribution from inelastic scattering by the sample was made using a wellestablished method $^{58}$, incorporating equations developed for total-scattering correlation functions to provide consistent definitions ${ }^{59}$.

The total-scattering structure factor $F(Q)$ measured in the absolute units of barn per steradian per atom is defined as follows:

$$
F(Q)=\sum_{\alpha \leq \beta}\left(2-\delta_{\alpha \beta}\right) c_{\alpha} c_{\beta} b_{\alpha} b_{\beta}\left[A_{\alpha \beta}(Q)-1\right]
$$

Where $c_{\alpha}$ and $c_{\beta}$ are the concentrations of atom type $\alpha$ and $\beta ; b_{\alpha}$ and $b_{\beta}$ are their corresponding neutron scattering lengths; $\delta_{\alpha \beta}$ is the Kronecker delta function to avoid double-counting interactions between like-atom pairs; $A \alpha_{\beta}(Q)$ are the Faber-Ziman partial structure factors.

Structure factor $S(Q)$ was obtained by normalizing $F(Q)$ :

$$
S(Q)=1+\frac{F(Q)}{\left(\sum_{\alpha} c_{\alpha} b_{\alpha}\right)^{2}}
$$

Where $\sum_{\alpha} c_{\alpha} b_{\alpha}$ is material scattering length

There is a negative swing at the low- $Q$ region in polymer $S(Q)$ in Fig. 4a. This is because the scattering length of the polymer is very small, due to the negative incoherent scattering length of $\mathrm{H}$, which then amplifies the negative peak in the low- $Q$ region of $F(Q)$ during normalization. This negative swing is thus not shown in Fig. 4a. This anomaly can be avoided if deuterated specimens are used, but was avoided in this study because of its affect on the dynamics of the setting process.

The total radial distribution function $G(r)$ is obtained by the direct Fourier transform of $F(Q)$ :

$$
G(r)=\frac{1}{(2 \pi)^{3} \rho} \int_{0}^{\infty} 4 \pi Q^{2} F(Q) \frac{\sin (Q r)}{Q r} d Q
$$

where $\rho$ is the atomic density of the material.

As the samples are sealed during measurements, trends in $S(Q)$ and $G(r)$ for the polymer are consistent with a fixed hydrogen content. Due to the effect of high hydrogen content of the GIC, conventional pair-distribution analysis of $G(r)$ was replaced by differencing (Fig. 4 f).

\section{References}

1. United Nations Environment Programme (UNEP). The Global Mercury Assessment 2013: Sources, Emissions, Releases and Environmental Transport (UNEP Chemicals Branch, 2013).

2. BIO Intelligence Service. Study on the potential for reducing mercury pollution from dental amalgam and batteries, Final report prepared for the European Commission-DG ENV (2012), Contract Number 07.0307/2011/59114/SER/C3.

3. Wilson, A. D. \& Kent, B. E. A new translucent cement for dentistry. The glass ionomer cement. Br. Dent. J. 15, 133-135 (1972).

4. Crisp, S. \& Wilson, A. D. Reactions in glass ionomer cements: I. decomposition of the powder. J. Dent. Res. 53, 1408-1413 (1974).

5. Crisp, S. \& Wilson, A. D. Reactions in glass ionomer cements: II. an infrared spectroscopic study. J. Dent. Res. 53, 1414-1419 (1974).

6. McLean, J. W. \& Wilson, A. D. The clinical development of the glass-ionomer cement. II. some clinical applications. Aus. Dent. J. 22, 120-127 (1977).

7. Nicholson, J. W. Chemistry of glass-ionomer cements: a review. Biomater 19, 485-494 (1998).

8. Davidson, C. L. Advances in glass-ionomer cements. J. Appl. Oral. Sci. 14, 3-9 (2006).

9. International Organization Standardization. ISO 7489. Dental glass polyalkenoate cements. Genebra; 1986; ICS:11.060.10.

10. Atmeh, A. R., Chong, E. Z., Richard, G., Festy, F. \& Watson, T. F. Dentin-cement interfacial interaction: calcium silicates and polyalkenoates. J. Dent. Res. 91, 454-459 (2012)
11. Ten Cate, J. M. \& van Duinen, R. N. B. Hypermineralization of dentinal lesions adjacent to glass-ionomer cement restorations. J. Dent. Res. 74, 1266-1271 (1995).

12. Lloyd, C. H. \& Mitchell, L. The fracture toughness of tooth coloured restorative materials. J. Oral. Rehabil. 11, 257-272 (1984).

13. Tian, K. V. et al. Qualitative assessment of microstructure and Hertzian indentation failure in biocompatible glass ionomer cements. J. Mater. Sci. Mater. Med. 23, 677-685 (2012).

14. Kovarik, R. E., Haubenreich, J. E. \& Gore, D. Glass ionomer cements: a review of composition, chemistry, and biocompatibility as a dental and medical implant material. J. Long Term Eff. Med. Implants 15, 655-671 (2005).

15. Jonck, L. M., Grobbelaar, C. J. \& Strating, H. Biological evaluation of glass-ionomer cement (Ketac-0) as an interface material in total joint replacement. A screening test. Clin. Mater. 4, 201-224 (1989).

16. Bauer, M., Pytel, J., Vona, I. \& Gerlinger, I. Combination of ionomer cement and bone graft for ossicular reconstruction. Eur. Arch. Otorhinolaryngol. 264, 1267-1273 (2007).

17. Hatton, P.V., Hurrell-Gillingham, K. \& Brook, I. M. Biocompatibility of glass-ionomer bone cements. J. Dent. 34, 598-601 (2006).

18. Hertzberg, R. W., Vinci, R. P. \& Hertzberg, J. L. Deformation and Fracture Mechanics of Engineering Materials (Wiley5th edn, 2012).

19. Ashby, M. Materials Selection in Mechanical Design (ButterworthHeinemann3rd edn, 1999).

20. Ritchie, R. O. The conflicts between strength and toughness. Nat. Mater. 10, 817-822 (2011).

21. Wondraczek, L. et al. Towards ultrastrong glasses. Adv. Mater. 23, 4578-4586 (2011).

22. Greaves, G. N., Greer, A. L., Lakes, R. S. \& Rouxel, T. Poisson's ratio and modern materials. Nat. Mater. 10, 823-837 (2011).

23. Lewandowski, J. J., Wang, W. H. \& Greer, A. L. Intrinsic plasticity or brittleness of metallic glasses. Phil. Mag. Lett. 85, 77-87 (2005).

24. Akinmade, A. O. \& Nicholson, J. W. Poisson's ratio of glass-polyalkenoate ("glass-ionomer") cements determined by an ultrasonic pulse method. J. Mater. Sci. Mater. Med. 6, 483-485 (1995).

25. Davies, R. A.. et al. Geometric, electronic and elastic properties of dental silver amalgam $\gamma-\left(\mathrm{Ag}_{3} \mathrm{Sn}\right), \gamma_{1}-\left(\mathrm{Ag}_{2} \mathrm{Hg}_{3}\right), \gamma_{2}-\left(\mathrm{Sn}_{8} \mathrm{Hg}\right)$ phases, comparison of experiment and theory. Intermetallics 18, 756-760 (2010).

26. Greaves, G. N. et al. Glancing-angle x-ray absorption spectroscopy of corroded borosilicate glass surfaces containing uranium. J. Am. Chem. Soc. 111, 4313-4324 (1989).

27. Xue, X. Y. \& Kanzaki, M. Al coordination and water speciation in hydrous aluminosilicate glasses: direct evidence from high-resolution heteronuclear ${ }^{1} \mathrm{H}-{ }^{27} \mathrm{Al}$ correlation NMR. Solid State Nucl. Magn. Reson. 31, 10-27 (2007).

28. Zainuddin, N., Karpukhina, N., Hill, R. G. \& Law, R. V. A long-term study on the setting reaction of glass ionomer cements by ${ }^{27} \mathrm{Al}$ MAS-NMR spectroscopy. Dent. Mater. 25, 290-295 (2009).

29. Acbas, G., Niessen, K. A., Snell, E. H. \& Markelz, A. G. Optical measurements of long-range protein vibrations. Nat. Commun. 5, 3076 (2014).

30. Averett, D., Cicerone, M. T., Douglas, J. F. \& de Pablo, J. J. Fast relaxation and elasticity-related properties of trehalose-glycerol mixtures. Soft Matter 8, 4936-4945 (2012).

31. $\mathrm{Hu}, \mathrm{W}$. et al. Optically enhanced coherent transport in $\mathrm{YBa}_{2} \mathrm{Cu}_{3} \mathrm{O}_{6.5}$ by ultrafast redistribution of interlayer coupling. Nat. Mater. 13, 705-711 (2014).

32. Grossman, M. et al. Correlated structural kinetics and retarded solvent dynamics at the metalloprotease active site. Nat. Struct. Mol. Biol. 18, 1102-1108 (2011).

33. McIntosh, A. I., Yang, B., Goldup, S. M., Watkinson, M. \& Donnan, R. S. Terahertz spectroscopy: a powerful new tool for the chemical sciences? Chem. Soc. Rev. 41, 2072-2082 (2012).

34. Mayers, J. Quantum effects in deep inelastic neutron scattering. Phys. Rev. B 41, 41-51 (1990).

35. Bowron, D. T. et al. NIMROD: the near and intermediate range order diffractometer of the ISIS second target station. Rev. Sci. Instrum. 81, 033905 (2010).

36. Stamboulis, A., Law, R. V. \& Hill, R. G. Characterisation of commercial ionomer cements using magic angle nuclear magnetic resonance (MAS-NMR). Biomater 25, 3907-3913 (2004).

37. Hill, R. G. \& Wood, D. Apatite-mullite glass ceramics. J. Mater. Sci. Mater. Med. 6, 311-318 (1995).

38. James, P. F. in Glasses and Glass-Ceramics. (ed. Lewis, M. H.) (Chapman and Hall, 1989).

39. Wasson, E. A. \& Nicholson, J. W. A study of the relationship between setting chemistry and properties of modified glass polyalkenoate cements. Br. Polym. J. 23, 179-183 (1990).

40. Pedersen, M. T. et al. Phase separation in an ionomer glass: insight from calorimetry and phase transitions. J. Non-Cryst. Solids 415, 24-29 (2015). 
41. Yue, Y. Z. \& Angell, C. A. Clarifying the glass-transition behaviour of water by comparison with hyperquenched inorganic glasses. Nature 427, 717-720 (2004).

42. Greaves, G. N. \& Sen, S. Inorganic glasses, glass-forming liquids and amorphizing solids. Adv. Phys. 56, 1-166 (2007).

43. Ryder, M. R., Civalleri, B., Bennett, T. D., Henke, S. \& Rudić, S. Identifying the role of terahertz vibrations in metal-organic frameworks: from gate-opening phenomenon to shear-driven structural destabilization. Phys. Rev. Lett. 113, 215502 (2014).

44. Greaves, G. N., Meneau, F., Majérus, O., Jones, D. G. \& Taylor, J. Identifying the vibrations that destabilize crystals and characterizes the glassy state. Science 308, 1299-1302 (2005).

45. Prentice, P. The influence of molecular weight on the fracture of glassy polymers. J. Mater. Sci. 20, 1445-1454 (1985).

46. Goldman, M. Fracture properties of composite and glass ionomer dental restorative materials. J. Biomed. Mater. Res. 19, 771-783 (1985).

47. Lloyd, C. H. \& Adamson, M. The development of fracture toughness and fracture strength in posterior restorative materials. Dent. Mater. 3, 225-231 (1987).

48. Hill, R. G., Wilson, A. D. \& Warrens, C. P. The influence of poly(acrylic acid) molecular weight on the fracture toughness of glass-ionomer cements. J. Mater. Sci. 24, 363-371 (1989).

49. Griffin, S. \& Hill, R. Influence of poly(acrylic acid) molar mass on the fracture properties of glass polyalkenoate cements. J. Mater Sci. 33, 5383-5396 (1998).

50. Seel, A. G., Sartbaeva, A., Mayers, J., Ramirez-Cuesta, A. J. \& Edwards, P. P. Neutron Compton scattering investigation of sodium hydride: From bulk material to encapsulated nanoparticulates in amorphous silica gel. J. Chem. Phys. 134, 114511 (2011).

51. Mayers, J. \& Reiter, G. The VESUVIO electron volt neutron spectrometer. Meas. Sci. Technol. 23, 045902 (2012).

52. Zanatta, M. et al. Structural evolution and medium range order in permanently densified vitreous $\mathrm{SiO}_{2}$. Phys. Rev. Lett. 112, 045501 (2014).

53. Moshaverinia, A., Roohpour, N., Chee, W. W. L. \& Schricker, S. R. A review of powder modifications in conventional glass-ionomer dental cements. J. Mater. Chem. 21, 1319-1328 (2011).

54. Mayers, J., Fielding, A. L. \& Senesi, R. Multiple scattering in deep inelastic neutron scattering: Monte Carlo simulations and experiments at the ISIS eVS inverse geometry spectrometer. Nucl. Instrum. Methods Phys. Rev. A 481, 454-463 (2002).

55. Krzystyniak, M., Richards, S. E., Seel, A. G. \& Fernandez-Alonso, F. Massselective neutron spectroscopy of lithium hydride and deuteride: Experimental assessment of the harmonic and impulse approximations. Phys. Rev. B 88, 184304 (2013)

56. Romanelli, G. et al. Direct measurement of competing quantum effects on the kinetic energy of heavy water upon melting. J. Phys. Chem. Lett. 4, 3251-3256 (2013).

57. Soper, A. K., Gudrun, N., \& Gudrun, X: programs for correcting raw neutron and X-ray diffraction data to differential scattering cross section. Rutherford Appleton Laboratory Technical report: RAL-TR-2011-013 https:// epubs.stfc.ac.uk/work/56240 (2011).

58. Soper, A. K. The radial distribution functions of water as derived from radiation total scattering experiments: is there anything we can say for sure? ISRN. Phys. Chem 2013, 279463 (2013).

59. Keen, D. A. A comparison of various commonly used correlation functions for describing total scattering. J. Appl. Cryst. 34, 172-177 (2001).
60. Roberts, J. C., Powers, J. M. \& Craig, R. G. Fracture toughness and critical strain energy release rate of dental amalgam. J. Mater. Sci. 13, 965-971 (1978).

61. Kinney, J. H., Marshall, S. J. \& Marshall, G. W. The mechanical properties of human dentin: a critical review and re-evaluation of the dental literature. Crit. Rev. Oral Biol. Med. 14, 13-29 (2003).

62. Toparli, M. \& Koksal, N. S. Hardness and yield strength of dentin from simulated nanoindentation tests. Comput. Meth. Prog. Biomed 77, 253-257 (2005).

\section{Acknowledgements}

We acknowledge support of the neutron experiments from ISIS Pulsed Neutron and Muon facility (Science \& Technology Facilities Council, UK). J. Khaliq is thanked for the TEM imaging and A.I. McIntosh and S. Sadiq for preliminary THz test runs. D. Setiadi, F. Fernandez-Alonso, A. Seel, A. Taylor, C. Andreani and R. Senesi are thanked for helpful discussions. Z. Zhou and W. Chen for graphics visualization. K.V.T. and C.D.-N. are grateful for financial support from the TÁMOP-4.2.1/B-09/1/KMR-20100001_Hungary, R.S.D. for the EPSRC: Selective activation of chemical bonds EP/I014845, G.A.C. and D.-C.F. to the Royal Society (IE20096), G.A.C. to the Engineering and Physical Sciences Research Council (EP/H030077/1 and EP/H030077/2) and G.N.G. to Sidney Sussex College, Cambridge (Visiting Fellowship) and Wuhan University of Technology (Strategic Scientist).

\section{Author contributions}

B.Y. and G.A.C. carried out TEM measurements. Y.Z.Y., K.V.T. and G.N.G. conducted the DSC characterizations and analysed the data with G.A.C. B.Y., R.S.D. and G.A.C. carried out the $\mathrm{THz}$ measurements and analysed data with K.V.T. and D.-C.F. K.V.T., J.M. and G.A.C. performed the NCS experiments and data analysis. C.D.-N. and J.W.N. also participated in the experiments and G.N.G. was involved in the data analysis. K.V.T., D.T.B., G.A.C. and G.N.G. did neutron diffraction measurements and data analysis with A.L.G. involved in the data analysis. K.V.T., G.A.C. and G.N.G. assembled the figures and wrote the manuscript with A.L.G. providing editorial assistance, and Y.Z.Y. was involved with sections related to DSC.

\section{Additional information}

Supplementary Information accompanies this paper at http://www.nature.com/ naturecommunications

Competing financial interests: The authors declare no competing financial interests.

Reprints and permission information is available online at http://npg.nature.com/ reprintsandpermissions/

How to cite this article: Tian, K. V. et al. Atomic and vibrational origins of mechanical toughness in bioactive cement during setting. Nat. Commun. 6:8631 doi: 10.1038/ncomms9631 (2015).

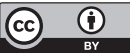

This work is licensed under a Creative Commons Attribution 4.0 International License. The images or other third party material in this article are included in the article's Creative Commons license, unless indicated otherwise in the credit line; if the material is not included under the Creative Commons license, users will need to obtain permission from the license holder to reproduce the material. To view a copy of this license, visit http://creativecommons.org/licenses/by/4.0/ 